\section{§. 15.}

Man wird nicht erwarten, dafs die Formel des \$. 13 durchaus mit den Beobachtungen übereinstimmen werde, aber so viel ist doch gewifs, dafs man mit den Beobachtungen ubereinstimmende Resultate erhalten werde, die Refractionen am Horizonte ausgenommen, wenn man die Coefficienten aus Beubachtungen der Refractionen selbst bestimmen will. Um doch wenigstens Ein Beispiel zu geben, so wollen wir die Rechnung mit einer in Bessels Fund. Astr. angeführten Beobachtung Svanberg's in Lappland vergleichen. Dieser beohachtete an $13^{\text {ten }}$ Dec. 1802 die Zenithdistanz des oberen Sonnenrandes $=89^{\circ} 43^{\prime} 45^{\prime \prime} 7$ und die Refraction $=37^{\prime} 47^{\prime \prime} 7$ lei einer Temperatur $-13,2 \mathrm{C}$. und einer Barometerhöhe von 0,73156 Metern. Daraus berechnet sich die Refraction $=37^{\prime} 51^{\prime \prime}$, welches von der Beobachtung nur 3"3 abweicht.

Aher solehe Uebereinstimmung findet nicht allenthalben statt, namentlich erhält man weit gröfsere Differenzen, wenn man die Rechnung mit den in Bessels Fund. Astr. angelührten Beob. achtungen Bradleys xergleicht, wo die berechneten Refractionen sämmtlich zu grofs ausfallen. Ein Theil des Fehlers könnte hier auf den Werth von $k$ fallen, welcher in Bessels angeführter Schrift ohngefähr um 1" kleiner angegehen wird als der oben, angefübrte. Da indessen hierdurch der Fehler nicht vollkommen gehohen wird, so mufs zu einer Aenderung der Coefficienten in der Formel \$.13 geschritten werden, welches Geschäft mit vieler Mühe verknüpft ist, die ich nicht ïber mich nehmen konnte, da mir dor nöthige Beistand mangelte. Aus diesen Grunde habe ich es auch unterlassen, Refractionstafeln zu berechnen, zumal da ich hiermit nichts über die Besselsche Arbeit hätte gewinnen können.

Was aber jetat in Bezug auf die Theorie der astronomischen Strahlenbrechung geleistet worden ist, lifst, so wie auch die Besselschen Refractionstafeln, in der practischen Astrononie nur Anwendung bis etwa $80^{\circ}$ oder $86^{\circ}$ scheiubarer Zenithdistanz zu. Dieses glaube ich durch die $\$ .4$ bis 9 auf dem einfachsten und sichersten Wege errcicht zu haben.

Weimar 1837.

$$
\text { Fr. Wr. Barfufs. }
$$

\title{
Ueber die Bahn des Doppelsterns 3062 Struve.
}

(Im Sternbilde der Cassiopeja, $\alpha=23^{\circ} 57^{\prime} 1, \delta=+57^{\circ} 28$.)

Dieses telescopische Sternenpaar hat seit Herschel bereits mehr als einen halben Umlauf vollendet und ist deshalb wohl geeignet, die noch sehr geringe Zahl derjenigen Sternsysteme, deren Elemente uns einigernafsen bekannt sind, zu vermehren. Da die Zahl der vorhandenen Beobachtungen kaum grölser ist als die, welche die Theoric absolut erfordert, so konnte die Darstellung derselben verhältuifsmälsig sehr genau sein, ohne dafs deshall auf einen bedeutenden Grad der Sicherbeit in den Elementen geschlossen werden kann. Diese Beobachtungen sind:

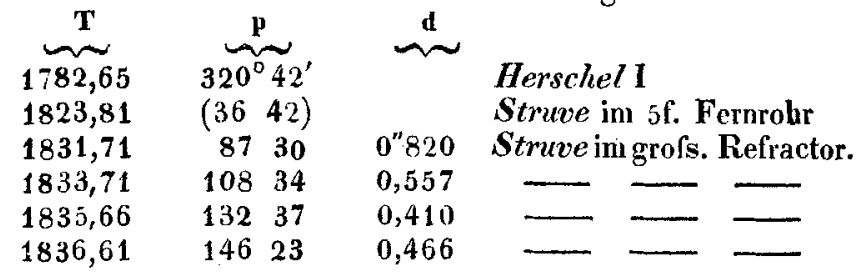

Die nur auf Schätzung beruhende Position von 1823,81 ist bei der definitiven Rechnung nicht mit hinzugezogen wordes. Die Elemente sind die folgenden:

$$
\begin{array}{ll}
\text { Halbe grofse Axe } & =a=1^{\prime \prime} 0033 \\
\text { Excentricität } & =e=0,53169=\sin 32^{\circ} 7^{\prime} 2 \\
\text { Abst. des Perihels vom } \delta & =\lambda=36^{\circ} 31^{\prime} 2 \\
\text { Neigung } & =\gamma=2531,6 \\
\text { Aufsteigender Knoten } & =\delta=12510,0 \\
\text { Umlaufszeit } & =84,5140 \mathrm{Jahre} . \\
\text { Zeit des Perihels } & =1837,427 .
\end{array}
$$

Damit wird erhalten :

$$
\begin{aligned}
& \frac{T}{1782} \\
& 1782,65 \\
& \overbrace{320^{\circ}}^{p} \\
& \overbrace{1 " 4187}^{d} \\
& \underbrace{\Delta \mathbf{p}}_{+23^{\prime}} \\
& \underline{\Delta d} \\
& 1823,81 \\
& 4034,8 \\
& 0,9017 \\
& 0,6114 \\
& (-231,8) \\
& -77,0+0 " 208
\end{aligned}
$$

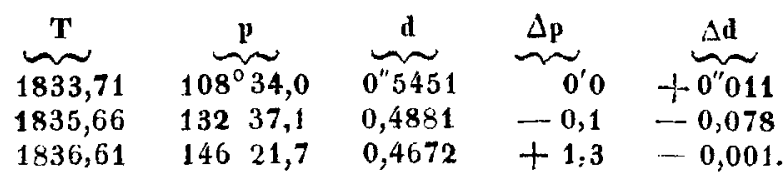

Da möglicherweise zwischen 1782 und 1823 nicht $76^{\circ}$ sondern $360^{\circ}+76^{\circ}$ zurückgelegt 'seyn konnten, so versuchte ich auch diese Hypothese. Hier ergab sich aber, wie zu erwarten war, ein Perihel auf der entgegengesetzten Seite, und für die beiden erwähnten Epochen so geringe Distanzen, da's weder Herschel (der doch die Distanz $=1$ Diameter setzt) noch Struve im 5f. Fernroht ihn hätten doppelt erblicken können.

Wir keunen also jetzt einen Doppelstern der bei ciner Umlaufszeit wie Uranus eine mittlere Distanz von nur 1" zeigt, und bei dem das Product $\pi \sqrt[3]{ } M$, oder die mit der Cubikwurzel der Mas=s multiplicirte Parallaxe 0 "0521 beträgt.

$\begin{array}{rlrl}1838,0 . & 168^{\circ} & 8,2 & 0^{\prime \prime} 449 \text { (Minimum) } \\ 5 . & 184 & 27,2 & 0,452 \\ 1839,0 . & 200 & 14,3 & 0,463 \\ 5 . & 214 & 38,5 & 0,484 \\ 1840,0 . & 227 & 14,4 & 0,520 \\ 5 . & 237 & 59,1 & 0,565 \\ 1841,0 . & 247 & 6,3 & 0,620 \\ 5 . & 254 & 48,0 & 0,676 \\ 1842,0 . & 261 & 28,2 & 0,729 \\ 5 . & 267 & 12,9 & 0,781 \\ 1843,0 . & 272 & 15,3 & 0,831 .\end{array}$

Bei dieser Gelegenheit bemerke ich noch, dafs die Umlaufszeit von $y$ Virgini's nicht, wie man bisher angenommen, 5 bis 6 Jahrhun. derte, sondern höchstens $180 \mathrm{Jahr}$ ist. Das Nähere darüber behalte ich mir vor, bis eine nene Reibe von Beobachtungen, die im Fruhjahr 1838 zu erwarten sind, ein noch fehlendes Berechvungsdatum gegeben halien wird. 